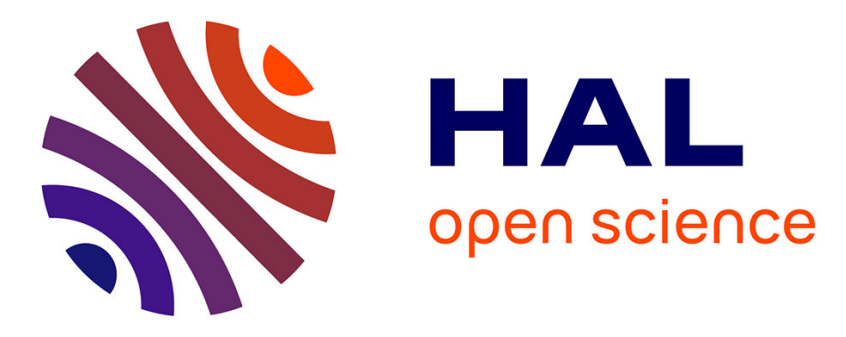

\title{
Considerations on the interaction between the press structure and the tool in the deep drawing simulation
}

\author{
G. Belingardi, A. Chiara, M. Fariello
}

\section{To cite this version:}

G. Belingardi, A. Chiara, M. Fariello. Considerations on the interaction between the press structure and the tool in the deep drawing simulation. Journal de Physique IV Proceedings, 1993, 03 (C7), pp.C7-1181-C7-1186. 10.1051/jp4:19937183 . jpa-00251815

\section{HAL Id: jpa-00251815 https://hal.science/jpa-00251815}

Submitted on 1 Jan 1993

HAL is a multi-disciplinary open access archive for the deposit and dissemination of scientific research documents, whether they are published or not. The documents may come from teaching and research institutions in France or abroad, or from public or private research centers.
L'archive ouverte pluridisciplinaire HAL, est destinée au dépôt et à la diffusion de documents scientifiques de niveau recherche, publiés ou non, émanant des établissements d'enseignement et de recherche français ou étrangers, des laboratoires publics ou privés. 


\title{
Considerations on the interaction between the press structure and the tool in the deep drawing simulation
}

\author{
G. BELINGARDI, A. CHIARA* and M. FARIELLO* \\ Mechanical Engineering Department, Politecnico of Torino, corso Duca degli Abruzzi 24, 10129 Torino, \\ Italy \\ * GEMS, Research \& Development Department, via Grugliasco 14, 10092 Beinasco, Torino, Italy
}

\begin{abstract}
The mathematical modelling of sheet metal forming operation is extended to include information about the particular tool and the particular machine that will be used in the "real" process. Particular attention is paid to the structure and to the operation of a mechanical press. For this type of process some control factors (parameters that the engineer can set during the fine tuning of the forming process on the machine) and some noise factors (parameters that are not under the control of the engineer) are individuated and a procedure to obtain a "robust" design of the process itself (i.e. a process optimized from the point of view of its low sensibility to the noise factors that are responsible of the loss of quality of the product) is outlined. The procedure is applied to the case of the deep drawing of an automotive rear fender, by the use of the OPTRIS finite element code, in order to demonstrate its capability .
\end{abstract}

\section{INTRODUCTION.}

The numerical simulation of sheet metal forming operation by means of the finite element method has recently reached a high level of quality, even in very large tridimensional industrial applications. Results are in very good accordance with experimental measurements both in terms of final deformed shapes, of strain field history and of punch force history $[1,2,3]$.

At present the finite element codes used to develop the simulations are based on the time integration of the dynamic non-linear equilibrium equations by explicit integration schemes [4,5]. This type of timeintegration procedure requires a very short time-step and the equation system has to be solved thousands of times but the total amount of time required for the complete simulation can result to be less (with large problem considerably less) than the amount of time required by the implicit integration scheme. Moreover the explicit integration scheme makes it possible to detect when and where wrinkles come out and to follow their evolution during the forming operation, while the implicit integration scheme is not capable to deal with this type of instability problems.

At this stage it becomes of interest to introduce into the mathematical modeling of the problem also some of the second order effects, that have been disregarded until now.

Until now, research activity was mainly devoted to a correct modeling of the elasto-plastic material behaviour and to reliable algorithm for the search and treatment of the contact points, for these reasons a simplified "ideal" deep drawing has been studied. A more accurate modeling of the process is now needed. It should be able to take into account information about the particular tool and the particular machine that will be used for the in-factory drawing. 
It is well known that in industrial plants two different types of press are used: the hydraulic press and the mechanical press, with large difference in terms of plant productivity, but even in terms of quality of the formed pieces.

After the design of the tool that will be used for the deep drawing operation, the forming process should be tuned working with the chosen press and making a sequence of progressive adjustments on a prototype of the tool. There are some parameters at disposal of the manufacturing engineer for this tuning. The knowledge of these parameters and of their effectiveness, not only from a general point of view but with reference to the particular case, is of great relevance in the practical tuning of the process.

The paper presents and discusses results obtained during the simulation of an automotive case, the deep drawing of a rear fender, where the a mechanical press with a constant clearance blank-holder was modeled in order to take into account the machine, the initial choice of the machine tuning parameters and the blank-holding force variation during the stamping process.

\section{OPTIMIZATION OF SHEET METAL FORMING PROCESS.}

It is well known that the deep drawing of a metallic sheet is essentially performed by the application of a properly chosen time history of boundary conditions to an elasto-plastic media so that the final configuration of the formed piece has the desired geometrical shape.

There are a number of different but not equivalent processes that satisfy the above definition, they are different one from the other by the particular sets of the process parameters that have been chosen by the process engineer, the resulting formed pieces are similar but not equal, although within the prescribed geometrical tolerances. Obviously also the quality of the formed pieces is different depending on the actual process: so that a forming process results to be more critical than another if fractures, surface oscillations or wrinkles are more likely to appear.

In industrial plants two different types of press are used: the hydraulic press and the mechanical press.

The first type of press has a hydraulic system that provides separately the force for the punch motion - that causes the deep drawing of the metal sheet - and the force for the blank-holder closure. The entity of this latter force can be controlled during the forming process but, generally, it remains constant during all the operation so that this is named "constant force blank-holder" (figure 1).

In the second type of press the blank-holding force is supplied by a mechanism and its entity results from the interaction between two compliances: on one side the compliance of the columns that act as stroke-end for the blank-holder and on the other side the compliance resulting from the sum of the blank-holder structure plus the sheet plus the die structure compliances (figure 2). In this way the blank-holding force

HYDRALLIC PRESS

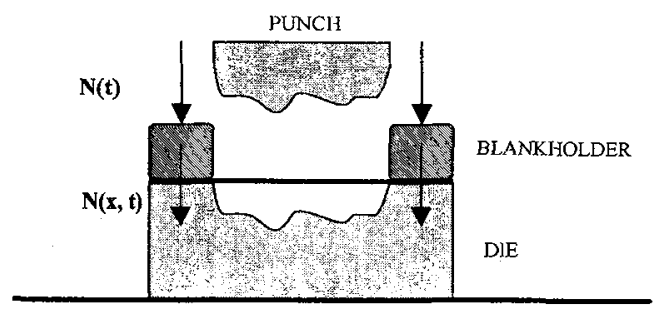

MECHANEC PRESS

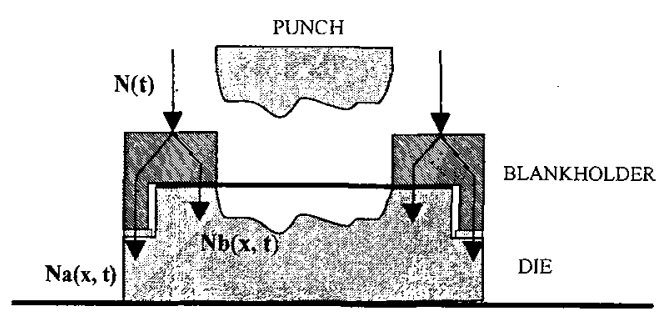

Fig. 1 Draft of hydraulic press blank-holder.

Fig. 2 Draft of mechanical press blank-holder. 
entity is not known a priori. In practice the blank-holding force should be chosen through a difficult tryand-error procedure. It should be pointed out that it changes from one sheet to another as the actual sheet thickness varies within the prescribed thickness tolerance, moreover it is subjected to change during the process itself as a consequence of the sheet thickness variation. Therefore this type of blank-holder is generally named "constant clearance blank-holder", although this is not completely correct.

The mechanical press is largely used in factories where large scale productions are required because of their higher productivity.

The in-factory tuning of the drawing process is devoted to adjust the machine parameters (for example the length of the blank-holder stroke ends that are strictly related to the blank-holding maximum force) and to modify the tool (for example by adding draw-beads or modifying their geometry) and the initial outer contour of the flat blank, that is always larger than the material strictly required for the final piece. In this way the process engineer can control the regularity of the material flow that follows the motion of the punch.

A fine tuning of the process is needed to adapt the "ideal" design to the "real" situation where a certain type of machine (with a defined although very high stiffness) equipped with a certain tool (constituted by that die, that punch and that blank-holder, each of them with a defined stiffness and some geometrical imperfections due to their manufacturing) are used to draw a certain metal sheet.

From a conceptual point of view it is possible to say that the control parameters do not affect directly the drawing process but their effect is somehow "filtered" by the real situation, in particular mainly by the flexibility of the blank-holder and at some extent also of the press structure.

At the end of this procedure, sometimes rather time-consuming, by an appropriate choice of some "control" parameters (figure 3), the real process is defined and even optimized.

It is clear now the advantage that can be obtained from the inclusion in the mathematical modeling also of information about the particular tool and the particular machine that will be used for the in-factory drawing and from the development of a large amount of the tuning work during the computer simulation phase, where a sensitivity analysis could also be performed in order to point out the more effective and the more convenient "control" parameters.

Moreover it can be realize that in the "real" drawing process there are also some "noise" factors (figure 3), i.e. some factors that are able to influence the quality of the formed piece (and sometimes even the piece feasibility) but are varying in an unpredictable (random) manner so that it is impossible or impractical to

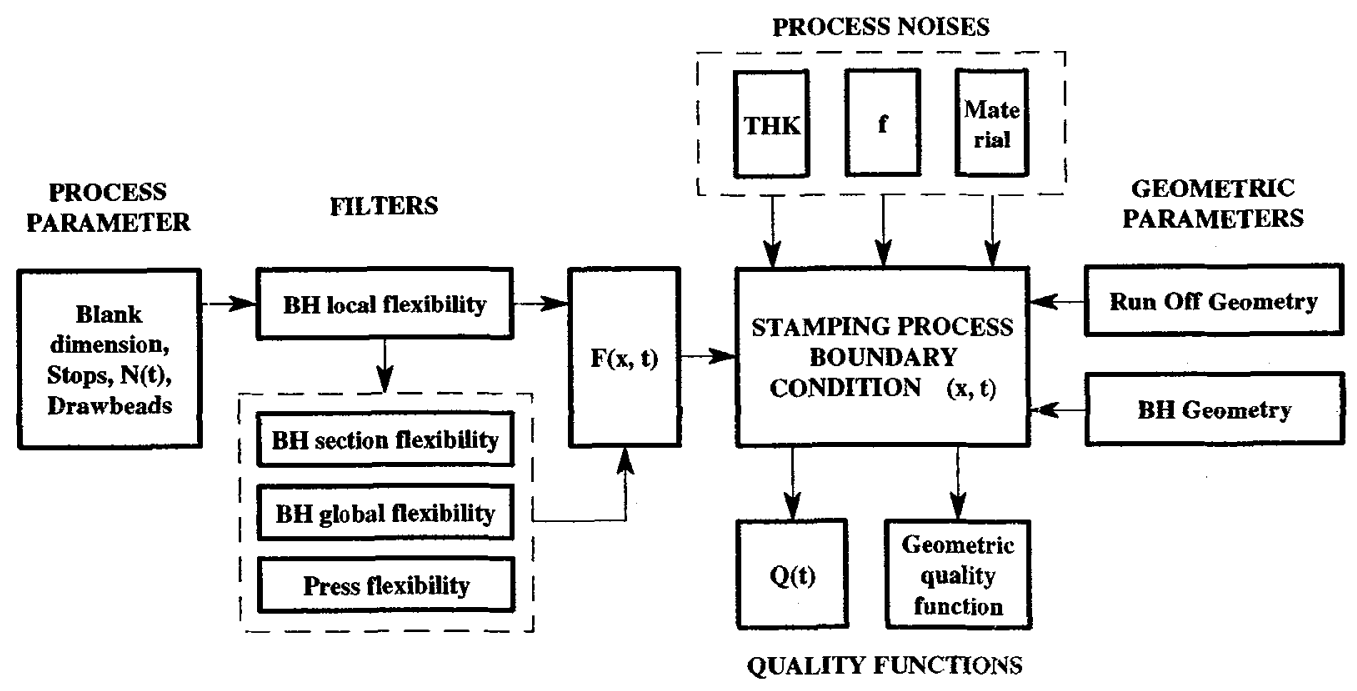

Fig. 3 Control parameters and noise factors in the deep drawing process. 


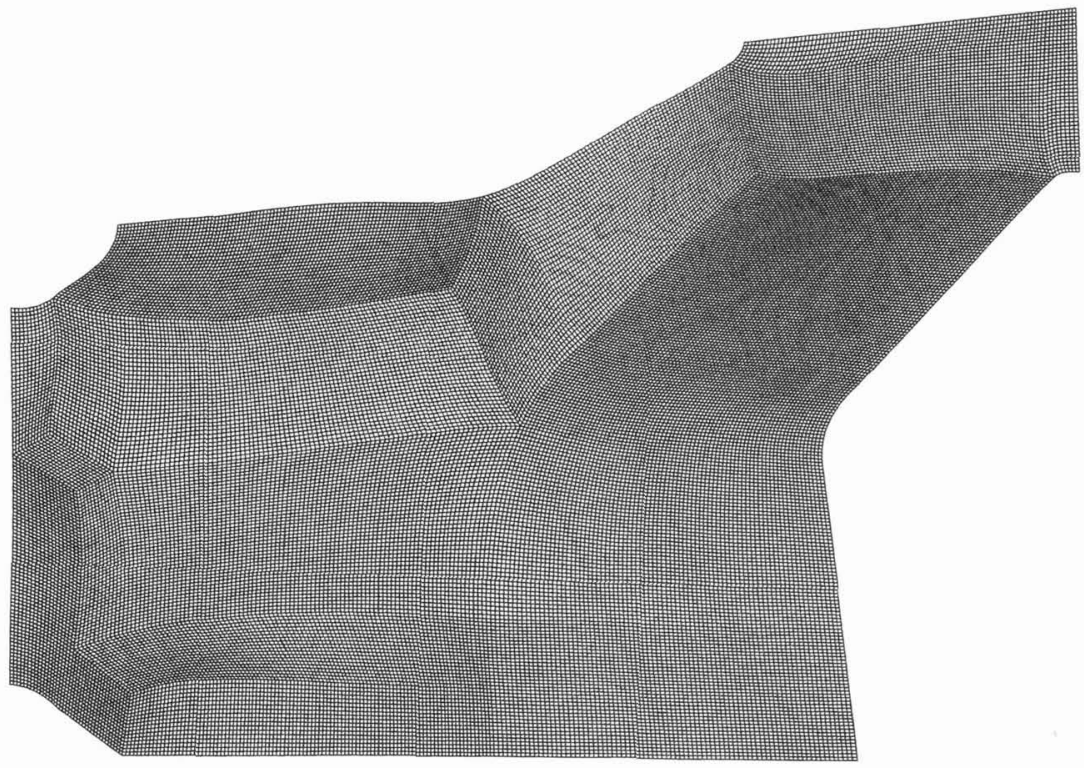

Fig. 4 The finite element mesh of the undeformed steel sheet.

modify the above mentioned drawing "control" parameters to prevent piece quality faults.

These noise factors are connected with the variability of the sheet thickness, of the mechanical characteristic of the material (for the example the yield strength), of the sheet physical state (previous cold working and residual stress field), of the sheet surface finishing and surface lubrication conditions (that influence the friction coefficient at the sliding interface between the sheet and the tool).

A good choice of the process control parameters could lead to a "robust" process, i.e. a process in which the response (in this case the quality of the formed pieces) is nearly insensible to the noise factors.

\section{THE AUTOMOTIVE REAR FENDER CASE.}

The piece, that has been used in the application, is the rear fender of a medium size car manufactured in Italy. Figure 4 shows the finite element model of the undeformed steel sheet $(40.000$ four node shell elements), prepared according to the requirement of the finite element code OPTRIS.

The blank-holder is modeled by eight nodes brick elements made of elastic material in order to take into account its actual flexibility, while the punch and the die, that are much more stiff, are modeled by four node shell elements made by "rigid" material.

To take into account the blank-holder flexibility is important from two points of view: at first in order to distribute in a realistic manner (the blank-holding pressure is actually not uniformly distributed) the blankholding force all over the portion of the sheet that is pinched between the die and the blank-holder itself. Secondly in order to absorb the local variation of the thickness of the sheet (this variation will otherwise cause a concentration of the blank-holding force on few localized points) where, following the material plastic flow, the increasing in thickness of the sheet is larger.

The draw-beads are included in the mathematical model through a special feature of OPTRIS that, using the results of a bidimensional analysis performed with the actual geometry, obtains the draw-bead effect on the sheet by imposing an equivalent force distribution.

The model of the tool includes also four bars that represent the columns that in the real press act as stroke end for the blank-holder when it is pushed down (see figure 2). The length and the stiffness of these bars are essential in defining the actual entity of the blank-holding force.

Results are collected both in terms of a sequence of deformed shapes, with particular reference to the final 


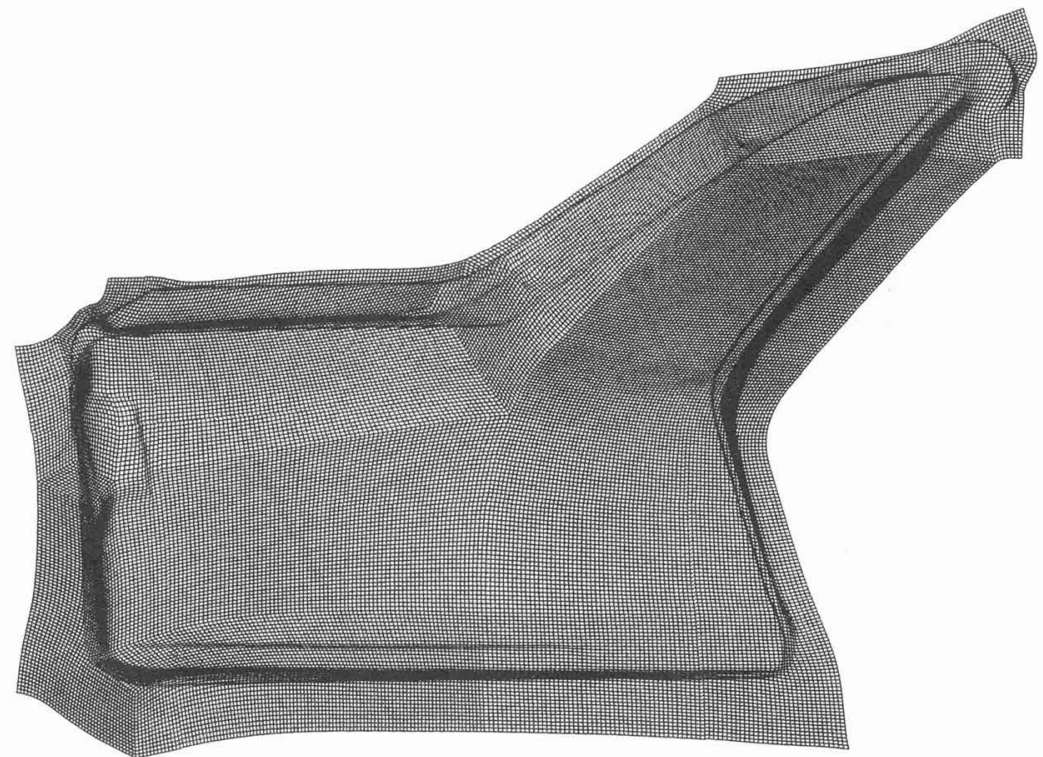

Fig. 5 A perspective view of the final deformed shape of the automotive rear fender.

configuration and to the resulting thickness distribution, and in terms of stress and strain fields history.

These results are analyzed utilizing the FICTURE post-processing code. In particular through the visualization of the deformed shape (see figure 5) it is, eventually, possible to recognize the presence of wrinkles and/or oscillation on the formed surface that are one of the critical aspects in the judgment on the success of the forming operation. On the other hand through the visualization of the thickness field it is, eventually, possible to recognize the presence of excessive thinning of the sheet that is symptom of necking and possible fracture. Finally reporting the strain field in the major-minor principal strains diagram and comparing the trajectory of each point through all the process with the material forming limit curve (FLD) it is possible, exactly in the same manner and with the same rules used in the drawing practice, to formulate a judgment on the formability of the piece (see figure 6).

In the present case the whole drawing simulation was repeated examining different values of the noise factors: the friction coefficient, that affects the sliding motion of the sheet over the tool surfaces, and the

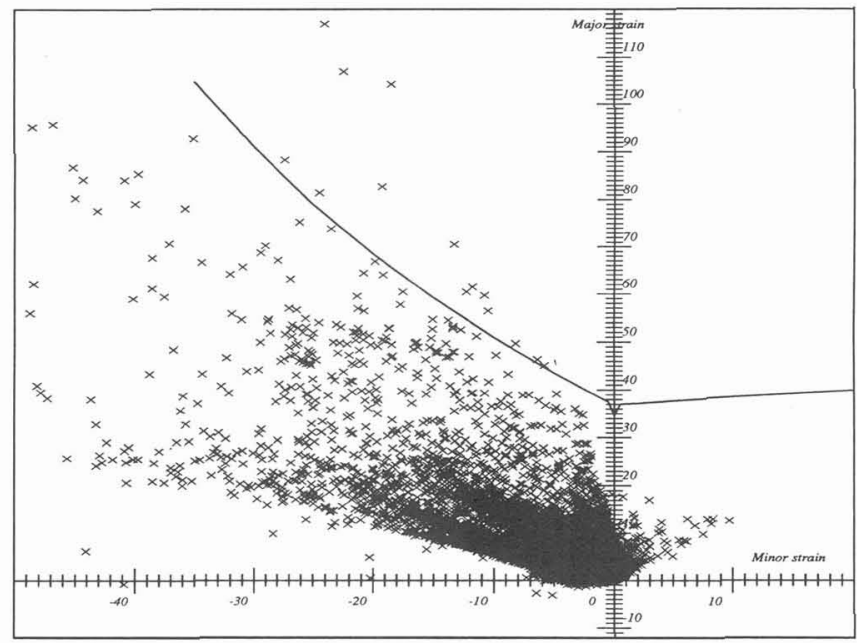

Fig. 6 Analysis of the principal strains field and comparison with the material limit forming diagram. 
thickness of the sheet. Both these noise factors have been considered homogeneous for all the model, although a random distribution of their value all over the sheet could be more realistic but too much expensive.

\section{CONCLUSIONS.}

The simulation of deep drawing operation by finite element code has been considered with reference to the inclusion of some second order effects into the mathematical model.

This is aimed to take into account during the simulation not only of the "ideal" elasto-plastic non-linear problem connected with the sheet deformation but also of the "real" condition of the drawing operation.

Taking advantage from the great level of quality already at disposal of the engineers from the point of view of the numerical simulation, it is now possible to transfer into the simulation phase also the study of the effect of the process "control" parameters. These parameters are currently used by the process engineer at the manufacturing phase of the project development to tune the drawing process. The simulation procedure is able to perform a sensibility analysis in order to identify the more effective and convenient parameters for the actual application.

The mathematical model is able to include also the "physical" filters that are essentially constituted by the tool structure and act on the effect of the control parameters in the "real" process.

Moreover in the "real" drawing process there are also some "noise" factors, i.e. some factors that are able to influence the quality of the formed piece (and sometimes even the piece feasibility) but are varying in an unpredictable (random) manner so that it is impossible or impractical to modify the above mentioned drawing "control" parameters to prevent piece quality faults.

A good choice of the process control parameters could lead to a "robust" process, i.e. a process in which the response (in this case the quality of the formed pieces) is nearly insensible to the noise factors.

Some results of the simulation of the deep drawing of an automotive rear fender, where the use of a mechanical press is taken into account, are shown to illustrate the outlined analysis procedure.

\section{ACKNOWLEDGMENTS.}

Authors wish to express their appreciation to the FIAT Auto Manufacturing Division for the permission of showing some results of the rear fender application.

One of the authors (G.B.) wishes also to acknowledge the financial support of the Italian Ministero dell'Università e della Ricerca Scientifica e Tecnologica, through $40 \%$ founds.

\section{REFERENCES.}

[1] Wagoner R., Zhou D. - "Analyzing sheet forming operations -recent numerical and experimental advances", proc. of NUMIFORM '92, Balkema, 1992

[2] Aberlenc F., Babeau J.L. - "OPTRIS and Ficture: industrial tools to modelize sheet metal forming", proc. of NUMIFORM '92, Balkema, 1992

[3] Belingardi G., Goglio L. - "Simulation of square box deep drawing using an explicit code: numerical and experimental results", proc. of 3rd Int. Conf. Computational Plasticity, Pineridge Press, 1992.

[4] Rebelo N., Nagtegaal J.C., Taylor L.M., Passman R. - "Comparison of implicit and explicit finite element methods in the simulation of metal forming processes", proc. of NUMIFORM ' 92 , Balkema, 1992

[5] Schweizerhof K., Hallquist J.O. - "Explicit integration schemes and contact formulations for thin sheet metal forming", proc. of "FE simulation of 3D sheet metal forming process" Int. Conf., VDI 1991. 